\title{
LOWER BOUNDS FOR EIGENVALUES OF HYPERSURFACE DIRAC OPERATORS
}

\author{
XIAO ZHANG
}

\section{Introduction}

Let $N$ be an $(n+1)$-dimensional Riemannian manifold and $M$ be an $n$-dimensional spin hypersurface in $N$. The choice of normal covector of $M$ yields a diagram

$$
\begin{array}{rll}
\operatorname{Spin}(n) & \stackrel{\hat{\alpha}}{\longrightarrow} & \operatorname{Spin}(n+1) \\
\rho_{1} \downarrow & & \downarrow \rho_{2} \\
S O(n) & \stackrel{\alpha}{\longrightarrow} & S O(n+1)
\end{array}
$$

such that $\rho_{2} \circ \hat{\alpha}=\alpha \circ \rho_{1}$.

Let $S$ be the spinor bundle of $N$. The above diagram implies $S$ is globallydefined along $M$. We call it hypersurface spinor bundle of $M$. Let $\widetilde{\nabla}$ be the LeviCivita connection of $N$, and $\nabla$ be its induced connection on $M$. We lift them to the hypersurface spinor bundle $S$, also denote them as $\widetilde{\nabla}$ and $\nabla$ respectively.

Denote $c$ the Clifford multiplication, the Dirac operator on $M$ defined by $\nabla$ on $S$ is the composition

$$
\Gamma(S) \stackrel{\nabla}{\longrightarrow} \Gamma\left(T^{*} M \otimes S\right) \stackrel{c}{\longrightarrow} \Gamma(S) .
$$

The hypersurface Dirac operator - denoted $\widetilde{D}-$ is defined by the second connection $\widetilde{\nabla}$ on $S$. Intrinsically, $\widetilde{D}$ is the composition

$$
\Gamma(S) \stackrel{\widetilde{\nabla}}{\longrightarrow} \Gamma\left(T^{*} M \otimes S\right) \stackrel{c}{\longrightarrow} \Gamma(S) .
$$

When $N$ is a Lorentzian manifold and $M$ is a spin spacelike hypersurface of $N$, this hypersurface Dirac operator was used to prove the Positive Energy Conjecture in general relativity by Witten [PT, W, Z]. Based on his proof of Positive Energy Conjecture with Schoen by using minimal surfaces [SY1, SY2], Yau asks what is the relation between minimal surfaces and (hypersurface) Dirac operators. Although its special significance is not very clear by this time, the hypersurface Dirac operator has shown its importance and potential application. We refer to [F2] (and references therein) on representation of surface in $R^{3}$ in terms of solutions of the hypersurface Dirac operator, and to [S1, S2, LS] on

Received October 7, 1997.

This work is partially supported by the Morningside Center of Mathematics, Chinese Academy of Sciences. 
applications in general relativity and in string theorey for hypersurface Dirac operators defined on higher-codimensional spin submanifolds (2-surfaces). It also provides a model for our attempt on the massive Seiberg-Witten theorey [YZ].

In this paper we study lower bounds for eigenvalues of a formally self-adjoint Dirac operator $\widetilde{D}^{*} \widetilde{D}$ on compact spin hypersurafce $M$ in a Riemannian manifold $N$. (It should be pointed out that, in general, $\widetilde{D}$ is not self-adjoint w.r.t. positive definite Hermitian metric on $S$ ). Our motivation is trying to understand the above question of Yau in this aspect. Let $\lambda$ be the eigenvalue of $\widetilde{D}^{*} \widetilde{D}$ with eigenspinor $\phi$, denote $R$ as scalar curvature of $M, H$ as the trace of the second fundamental form of $M$, we prove

$$
\lambda \geq \frac{1}{4} \sup _{a} \inf _{M}\left(\frac{R}{1+n a^{2}-2 a}-\frac{(n-1) H^{2}}{(1-n a)^{2}}\right),
$$

where $a$ is any real number, $a \neq \frac{1}{n}$ if $H \neq 0$. And

$$
\lambda \geq \frac{1}{4} \inf _{M}\left(R-(n-1) H^{2}\right)+\inf _{M}\left|Q_{\phi}\right|^{2},
$$

where $\left|Q_{\phi}\right|^{2}=\frac{1}{4} \sum_{i, j}\left(\mathcal{R} e\left(e^{i} \nabla_{j} \phi+e^{j} \nabla_{i} \phi, \phi /|\phi|^{2}\right)\right)^{2}$. We also study the manifolds where $\lambda$ achieves its minimum. Particulally, equality in (1.1) gives an Einstein metric on $M$ with constant mean curvature.

Eigenvalue estimate for Dirac operator on compact spin manifolds is interested both in mathematics and in physics. Vafa and Witten estimated some upper bounds for twistor Dirac operators, which are independent on the twisted bundles, and study their applications in physics [VW]. Let $D \phi=\lambda \phi$ on some compact spin manifold $M$, where $\lambda$ is the eigenvalue of $D$ with eigenspinor $\phi$. In 1963, Lichnerowitz first proved that

$$
\lambda^{2} \geq \frac{1}{4} \inf _{M} R,
$$

where $R$ is the scalar curvature of $M$ [L]. In 1980, Friedrich proved that, for $n \geq 2$,

$$
\lambda^{2} \geq \frac{n}{4(n-1)} \inf _{M} R
$$

[F1]. In 1984, Hijazi proved that, for $n \geq 3$,

$$
\lambda^{2} \geq \frac{n}{4(n-1)} \mu_{1}
$$

where $\mu_{1}$ is the first eigenvalue of Yamabe operator $\frac{4(n-1)}{n-2} \triangle+R$ [Hi1]. Equality in (1.3) gives a Ricci-flat metric, that in (1.4) or (1.5) gives an Einstern metric. In 
terms of some real symmetric endomorphism of (co)tangent bundle, he improved lower bounds by [Hi2]

$$
\begin{gathered}
\lambda^{2} \geq \inf _{M}\left(\frac{1}{4} R+\left|Q_{\phi}\right|^{2}\right), \\
\lambda^{2} \geq \frac{1}{4} \mu_{1}+\inf _{M}\left|Q_{\phi}\right|^{2} .
\end{gathered}
$$

We note while $M$ is a minimal spin hypersurface in $N,(1.1)$ reduces to (1.4) and (1.2) reduces to (1.6).

\section{Hypersurface Dirac operators}

Suppose $N$ is an $(n+1)$-dimensional Riemannian manifold and $M$ is an $n$ dimensional spin hypersurface in $N$. Let $S$ be the hypersurface spinor bundle of $M$. It is known [LM] that there exists a positive definite Hermitian metric on $S$ which satisfies, for any covector $v \in T^{*} N$,

$$
(v . \phi, v . \psi)=|v|^{2}(\phi, \psi) .
$$

This metric is globally-defined along $M$. The connection $\widetilde{\nabla}$ is compatible with the metric ( , ). Fix a point $p \in M$ and an orthonormal basis $\left\{e_{\alpha}\right\}$ of $T_{p} N$ with $e_{0}$ normal and $e_{i}$ tangent to $M$ such that $\left(\nabla_{i} e_{j}\right)_{p}=0,\left(\widetilde{\nabla}_{0} e_{j}\right)_{p}=0,1 \leq i \leq n$. Let $\left\{e^{\alpha}\right\}$ be the dual coframe. Then $\left(\widetilde{\nabla}_{i} e^{j}\right)_{p}=-h_{i j} e^{0},\left(\widetilde{\nabla}_{i} e^{0}\right)_{p}=h_{i j} e^{j}, 1 \leq$ $i, j \leq n$, where $h_{i j}=\left(\widetilde{\nabla}_{i} e_{0}, e_{j}\right)$ are the components of the second fundamental form at $p$, and we have

$$
\widetilde{\nabla}_{i}=\nabla_{i}+\frac{1}{2} h_{i j} e^{0} e^{j}
$$

By $(2.8),\left(e^{0} e^{j} \phi, \psi\right)=\left(\phi, e^{j} e^{0} \psi\right)$. Then at $p \in M$,

$$
\begin{aligned}
d(\phi, \psi) * e_{i}= & \left(\left(\widetilde{\nabla}_{i} \phi, \psi\right)+\left(\phi, \widetilde{\nabla}_{i} \psi\right)\right) * 1 \\
= & \left(\left(\nabla_{i} \phi, \psi\right)+\left(\phi, \nabla_{i} \psi\right)\right. \\
& \left.+\frac{1}{2} h_{i j}\left(e^{0} e^{j} \phi, \psi\right)+\frac{1}{2} h_{i j}\left(\phi, e^{0} e^{j} \psi\right)\right) * 1 \\
= & \left(\left(\nabla_{i} \phi, \psi\right)+\left(\phi, \nabla_{i} \psi\right)\right) * 1 .
\end{aligned}
$$

Hence the connection $\nabla$ is also compatible with the metric $(, \quad)$.

If $n$ is even, the hypersurface spinor bundle $S$ splits as a direct sum of positive and negative eigenspaces $S^{ \pm}$of the operator $*=e^{1} e^{2} \ldots e^{n}$ along $M$. The connection $\nabla$ preserves this splittings since it commutes with operator $*$, but the connection $\widetilde{\nabla}$ does not preserve this splittings. Since $* e^{0}=e^{0} *, * e^{i}=-e^{i} *$, we then have $e^{0} S^{ \pm}=S^{ \pm}, e^{i} S^{ \pm}=S^{\mp}$. Moreover, for any $\phi \in S^{+}, \psi \in S^{-}$, $(\phi, \psi)=(* \phi,-* \psi)=-(\phi, \psi)$, hence $(\phi, \psi)=0$. In the above orthonormal coframe $\left\{e^{i}\right\}$ of $M, D=e^{i} \nabla_{i}, \widetilde{D}=e^{i} \widetilde{\nabla}_{i}$. 
Lemma 2.1. For any $\phi \in \Gamma(S)$, we have

$$
\widetilde{D} \phi=D \phi+\frac{H}{2} e^{0} \phi,
$$

where $H=\sum h_{i i}$ is the mean curvature of $M$.

Proof. Since $h_{i j}=h_{j i}$, and $e^{i} e^{j}=-e^{j} e^{i}$ for $i \neq j$, then (2.9) gives

$$
\widetilde{D} \phi=e^{i} \widetilde{\nabla}_{i} \phi=e^{i} \nabla_{i} \phi+\frac{1}{2} h_{i j} e^{i} e^{0} e^{j} \phi=D \phi+\frac{H}{2} e^{0} \phi .
$$

Lemma 2.2. For any $\phi, \psi \in \Gamma(S)$, we have

$$
\begin{aligned}
d\left(e^{i} \phi, \psi\right) * e^{i} & =((D \phi, \psi)-(\phi, D \psi)) * 1 \\
& =\left((\widetilde{D} \phi, \psi)-\left(\phi,\left(\widetilde{D}-H e^{0}\right) \psi\right)\right) * 1 .
\end{aligned}
$$

Proof.

$$
\begin{aligned}
d\left(e^{i} \phi, \psi\right) * e^{i} & =\left(\left(e^{i} \nabla_{i} \phi, \psi\right)+\left(e^{i} \phi, \nabla_{i} \psi\right)\right) * 1 \\
& =((D \phi, \psi)-(\phi, D \psi)) * 1 \\
& =\left(\left(\widetilde{D} \phi-\frac{H}{2} e^{0} \phi, \psi\right)-\left(\phi, \widetilde{D} \psi-\frac{H}{2} e^{0} \psi\right)\right) * 1 \\
& =\left((\widetilde{D} \phi, \psi)-\left(\phi,\left(\widetilde{D}-H e^{0}\right) \psi\right)\right) * 1 .
\end{aligned}
$$

Corollary 2.1. $\quad D^{*}=D, \quad \widetilde{D}^{*}=\widetilde{D}-H e^{0}=D-\frac{H}{2} e^{0}$.

If $M$ is an even dimensional spin hypersurface, let $\Phi=\phi+\psi \in \Gamma\left(S^{+}\right) \oplus \Gamma\left(S^{-}\right)$ and $\tilde{\psi}=-e^{0} \psi$, then $\widetilde{D} \Phi=0$ is equivalent to the following equations

$$
D \phi=\frac{H}{2} \tilde{\psi}, \quad D \tilde{\psi}=\frac{H}{2} \phi .
$$

Physically, $\frac{H}{2}$ is interpreted as the mass of spinor $\phi+\tilde{\psi}$.

Proposition 2.1. If $M$ is an even dimensional spin hypersurface which is not minimal, then any 'half' spinor solution $\widetilde{D} \phi=0$ is trivial.

Proof. If there is such spinor $\phi \in \Gamma\left(S^{+}\right)$. Then

$$
0=\widetilde{D} \phi=D \phi+\frac{H}{2} e^{0} \phi \in \Gamma\left(S^{-}\right) \oplus \Gamma\left(S^{+}\right) .
$$

Hence $D \phi=0$ and $H e^{0} \phi=0$. By the assumption, there is an open set $\Omega$ such that $H \neq 0$ on $\Omega$, therefore $\phi=0$ on $\Omega$. Then the Unique Continuation Property gives that $\phi \equiv 0$. Similarly, there is no such nontrivial $\psi \in \Gamma\left(S^{-}\right)$. 
The well-known Lichnerowicz formula [L] gives

$$
D^{*} D=\nabla^{*} \nabla+\frac{R}{4}
$$

where $R$ is the scalar curvature of $M$.

For the hypersurface Dirac operator, we have the following Lichnerowicz formulas

Theorem 2.1. For any $\phi \in \Gamma(S)$,

$$
\begin{aligned}
& \widetilde{D}^{*} \widetilde{D} \phi=\nabla^{*} \nabla \phi+\frac{1}{4}\left(R+H^{2}\right) \phi-\frac{1}{2} e^{0} d H \phi-H e^{0} D \phi, \\
& \widetilde{D} \widetilde{D}^{*} \phi=\nabla^{*} \nabla \phi+\frac{1}{4}\left(R+H^{2}\right) \phi+\frac{1}{2} e^{0} d H \phi+H e^{0} D \phi,
\end{aligned}
$$

where $R$ and $H$ are scalar and mean curvatures of $M$ respectively.

Proof. Since

$$
\begin{aligned}
\nabla_{i}\left(e^{0} \phi\right) & =\left(\widetilde{\nabla}_{i}-\frac{1}{2} h_{i j} e^{0} e^{j}\right)\left(e^{0} \phi\right) \\
& =h_{i j} e^{j} \phi+e^{0} \widetilde{\nabla}_{i} \phi-\frac{1}{2} h_{i j} e^{j} \phi \\
& =e^{0}\left(\widetilde{\nabla}_{i}-\frac{1}{2} h_{i j} e^{0} e^{j}\right) \phi=e^{0} \nabla_{i} \phi,
\end{aligned}
$$

then the theorem follows from the Lemma 2.1 and (2.12).

Now we define two modified connections on $\Gamma(S)$ by

$$
\begin{aligned}
& \nabla_{i}^{l}=\nabla_{i}+\frac{H}{2} e^{0} e^{i}, \\
& \nabla_{i}^{r}=\nabla_{i}-\frac{H}{2} e^{0} e^{i} .
\end{aligned}
$$

It is from a straightforward computation that

$$
\begin{aligned}
d(\phi, \psi) * e_{i} & =\left(\left(\nabla_{i}^{l} \phi, \psi\right)+\left(\phi, \nabla_{i}^{l} \psi\right)\right) * 1, \\
& =\left(\left(\nabla_{i}^{r} \phi, \psi\right)+\left(\phi, \nabla_{i}^{r} \psi\right)\right) * 1 .
\end{aligned}
$$

Hence both $\nabla^{l}$ and $\nabla^{r}$ are compatible with the metric $(, \quad)$. Furthermore,

$$
\begin{aligned}
\nabla_{i}^{l} \nabla_{i}^{l} \phi & =\left(\nabla_{i}+\frac{H}{2} e^{0} e^{i}\right)\left(\nabla \phi+\frac{H}{2} e^{0} e^{i} \phi\right) \\
& =\nabla_{i} \nabla_{i} \phi-\frac{H^{2}}{4} \phi+H e^{0} e^{i} \nabla_{i} \phi+\frac{1}{2} \nabla_{i} H e^{0} e^{i} \phi, \\
\nabla_{i}^{r} \nabla_{i}^{r} \phi & =\left(\nabla_{i}-\frac{H}{2} e^{0} e^{i}\right)\left(\nabla \phi-\frac{H}{2} e^{0} e^{i} \phi\right) \\
& =\nabla_{i} \nabla_{i} \phi-\frac{H^{2}}{4} \phi-H e^{0} e^{i} \nabla_{i} \phi-\frac{1}{2} \nabla_{i} H e^{0} e^{i} \phi .
\end{aligned}
$$


We can now obtain

Theorem 2.2. For any $\phi \in \Gamma(S)$,

$$
\begin{aligned}
& \widetilde{D}^{*} \widetilde{D} \phi=\nabla^{l *} \nabla^{l} \phi+\frac{1}{4}\left(R-(n-1) H^{2}\right) \phi, \\
& \widetilde{D} \widetilde{D}^{*} \phi=\nabla^{r *} \nabla^{r} \phi+\frac{1}{4}\left(R-(n-1) H^{2}\right) \phi,
\end{aligned}
$$

where $R$ and $H$ are scalar and mean curvatures of $M$ respectively.

Proposition 2.2. Let $M \subset N$ is a non-minimal compact spin hypersurface. If its scalar curvature $R$ and mean curvature $H$ satisfy

$$
R \geq(n-1) H^{2}
$$

Then the equation

$$
\widetilde{D}^{*} \widetilde{D} \phi=0, \quad \text { or } \quad \widetilde{D} \widetilde{D}^{*} \phi=0
$$

has only trivial solution.

Proof. Any solution of either $\widetilde{D}^{*} \widetilde{D} \phi=0$ or $\widetilde{D} \widetilde{D}^{*} \phi=0$ satisfies either $\widetilde{D} \phi=0$, $\nabla^{l} \phi=0$ or $\widetilde{D}^{*} \phi=0, \nabla^{r} \phi=0$. We then obtain $d|\phi|^{2}=0, D \phi=0$ and $H e^{0} \phi=0$ in both cases. This implies $H \equiv 0$ and contradicts to our assumption.

\section{Lower bounds of Eigenvalues}

Since operators $\widetilde{D}^{*} \widetilde{D}$ and $\widetilde{D} \widetilde{D}^{*}$ are self-adjoint w.r.t. Hermitian inner product $($, ). They have real eigenvalues. Furthermore, the eigenvalues of above two operators are the same.

Theorem 3.1. Let $M \subset N$ be a compact spin hypersurface, and $\lambda$ be the eigenvalue of $\widetilde{D}^{*} \widetilde{D}$. Then

$$
\lambda \geq \frac{1}{4} \sup _{a} \inf _{M}\left(\frac{R}{1+n a^{2}-2 a}-\frac{(n-1) H^{2}}{(1-n a)^{2}}\right),
$$

where $a$ is any real number, $a \neq \frac{1}{n}$ if $H \neq 0$. If $\lambda$ achieves its minimun, $M$ must have constant Ricci and mean curvatures,

$$
R_{i j}=\frac{(n-1)\left(1+n a_{0}^{2}-2 a_{0}\right)^{2}}{\left(1-n a_{0}\right)^{4}} H^{2} \delta_{i j}
$$

with eigenvalue $\lambda=\frac{(n-1)^{2}}{4\left(1-n a_{0}\right)^{4}} H^{2}$, where $a_{0}$ is chosen such that the right side of (3.19) achieves its maximum. 
Proof. Define a modified covariant derivative on $\Gamma(S)$ by

$$
L_{i}=\nabla_{i}+b \frac{H}{2} e^{0} e^{i}+a e^{i} \widetilde{D}
$$

We have

$$
\begin{aligned}
\left|L_{i} \phi\right|^{2}= & \left|\nabla_{i} \phi\right|^{2}+\frac{b^{2} H^{2}}{4}|\phi|^{2}+a^{2}|\widetilde{D} \phi|^{2} \\
& -b H \mathcal{R} e\left(e^{0} e^{i} \nabla_{i} \phi, \phi\right)-2 a \mathcal{R} e\left(e^{i} \nabla_{i} \phi, \widetilde{D} \phi\right) \\
& -a b H \mathcal{R} e\left(e^{0} \phi, \widetilde{D} \phi\right) .
\end{aligned}
$$

Therefore

$$
\begin{aligned}
|L \phi|^{2}= & |\nabla \phi|^{2}+\frac{n b^{2} H^{2}}{4}|\phi|^{2}+n a^{2}|\widetilde{D} \phi|^{2} \\
& -b H \mathcal{R} e\left(e^{0} D \phi, \phi\right)-2 a \mathcal{R} e(D \phi, \widetilde{D} \phi) \\
& -n a b H \mathcal{R} e\left(e^{0} \phi, \widetilde{D} \phi\right) \\
= & |\nabla \phi|^{2}+(n a b-a-b) H \mathcal{R} e\left(e^{0} D \phi, \phi\right) \\
& +\left(n a^{2}-2 a\right)|\widetilde{D} \phi|^{2}+\frac{H^{2}}{4}\left(n b^{2}+2 a-2 n a b\right)|\phi|^{2}
\end{aligned}
$$

And

$$
\begin{aligned}
\int_{M}|\widetilde{D} \phi|^{2}= & \int_{M}|\nabla \phi|^{2}+\frac{1}{4}\left(R+H^{2}\right)|\phi|^{2}-H \mathcal{R} e\left(e^{0} D \phi, \phi\right) \\
= & \int_{M}|L \phi|^{2}-(n a b-a-b) H \mathcal{R} e\left(e^{0} D \phi, \phi\right) \\
& -\int_{M}\left(n a^{2}-2 a\right)|\widetilde{D} \phi|^{2}+\frac{H^{2}}{4}\left(n b^{2}+2 a-2 n a b\right)|\phi|^{2} \\
& +\int_{M} \frac{1}{4}\left(R+H^{2}\right)|\phi|^{2}-H \mathcal{R} e\left(e^{0} D \phi, \phi\right) \\
= & \int_{M}|L \phi|^{2}-(n a b-a-b+1) H \mathcal{R} e\left(e^{0} D \phi, \phi\right)+\frac{R}{4}|\phi|^{2} \\
& -\int_{M}\left(n a^{2}-2 a\right)|\widetilde{D} \phi|^{2}+\frac{H^{2}}{4}\left(n b^{2}+2 a-2 n a b-1\right)|\phi|^{2} .
\end{aligned}
$$

Take $b=\frac{1-a}{1-n a}$, we obtain

$$
\int_{M}|\widetilde{D} \phi|^{2}=\int_{M} \frac{|L \phi|^{2}}{1+n a^{2}-2 a}+\frac{1}{4}\left(\frac{R}{1+n a^{2}-2 a}-\frac{(n-1) H^{2}}{(1-n a)^{2}}\right)|\phi|^{2} .
$$

Hence the first part of the theorem follows. 
If $\lambda$ achieves its minimun, then $L_{i} \phi=0$. This implies

$$
\nabla_{i} \phi=-\frac{\widetilde{H}}{2} e^{0} e^{i} \phi, \quad D \phi=-\frac{n \widetilde{H}}{2} e^{0} \phi,
$$

where $\widetilde{H}=\frac{1+n a_{0}^{2}-2 a_{0}}{\left(1-n a_{0}\right)^{2}} H$. Obviously, $d|\phi|^{2}=0$. On the other hand,

$$
\begin{aligned}
\sum_{k, l} \frac{1}{4} R_{i j k l} e^{k} e^{l} \phi= & \left(\nabla_{j} \nabla_{i}-\nabla_{i} \nabla_{j}\right) \phi \\
= & \frac{1}{2}\left(\nabla_{i} \widetilde{H} e^{0} e^{j}-\nabla_{j} \widetilde{H} e^{0} e^{i}\right) \phi \\
& +\frac{\widetilde{H}}{2} e^{0}\left(e^{j} \nabla_{i} \phi-e^{i} \nabla_{j} \phi\right) .
\end{aligned}
$$

Thus

$$
\begin{aligned}
\frac{1}{2} \sum_{k} R_{i k} e^{k} \phi= & \frac{1}{4} \sum_{j, k, l} R_{i j k l} e^{j} e^{k} e^{l} \phi \\
= & \frac{n-2}{2} \nabla_{i} \widetilde{H} e^{0} \phi+\frac{(n-2) \widetilde{H}}{2} e^{0} \nabla_{i} \phi \\
& -\frac{1}{2} e^{0} e^{i} d \widetilde{H} \phi+\frac{n \widetilde{H}^{2}}{4} e^{i} \phi
\end{aligned}
$$

And

$$
\begin{aligned}
-\frac{1}{2} R \phi & =\frac{1}{2} \sum_{i, k} R_{i k} e^{i} e^{k} \phi \\
& =(n-1) d \widetilde{H} e^{0} \phi-\frac{n(n-1) \widetilde{H}^{2}}{2} \phi .
\end{aligned}
$$

Take the inner product of the above equality with $\phi$ and compare its real part, we obtain, $R=n(n-1) \widetilde{H}^{2}$. Hence $d H=0$. Thus,

$$
\sum_{j} R_{i j} e^{j} \phi=(n-1) \widetilde{H}^{2} e^{i} \phi
$$

Therefore

$$
R_{i j}=\frac{(n-1)\left(1+n a_{0}^{2}-2 a_{0}\right)^{2}}{\left(1-n a_{0}\right)^{4}} H^{2} \delta_{i j}
$$

and

$$
\lambda=\frac{(n-1)^{2}}{4\left(1-n a_{0}\right)^{4}} H^{2} .
$$

Remark 3.1. If $H=0$, we can choose $a=\frac{1}{n}$, then $\lambda \geq \frac{n}{4(n-1)} \inf _{M} R$. This was proved by Friedrich [F1]. 
Now we estimete eigenvalue of $\widetilde{D}^{*} \widetilde{D}$ following an argement of Hijazi [Hi2]. For any spinor $\phi$, we define the associated real symmetric biliner form $Q_{\phi}$ on the complement of its zero set by, for any tangent vector fields $X, Y$,

$$
Q_{\phi}(X, Y)=\frac{1}{2} \mathcal{R} e\left(X \nabla_{Y} \phi+Y \nabla_{X} \phi, \phi /|\phi|^{2}\right) .
$$

If $\phi$ is the eigenspinor of $\widetilde{D} * \widetilde{D}, Q_{\phi}$ is well-defined in the sense of distribution.

Let $\mathcal{T}$ be some real symmetric endomorphism of cotangent bundle $T^{*} M$. Choose local orthonormal coframe $e^{i}$, we can write $\mathcal{T}\left(e^{i}\right)=\sum_{j} t_{i j} e^{j}$ where $t_{i j}=t_{j i}$ are some real functions. Define a modified covariant derivative by

$$
\nabla_{i}^{t}=\nabla_{i}+\mathcal{T}\left(e^{i}\right)+\frac{H}{2} e^{0} e^{i}
$$

on $\Gamma(S)$. Now it is easy to derive

$$
d(\phi, \psi) * e^{i}=\left(\left(\nabla_{i}^{t} \phi, \psi\right)+\left(\phi, \nabla_{i}^{t} \psi\right)\right) * 1 .
$$

Hence $\nabla^{t}$ is compatable with metric $($,$) .$

A straightforward computation gives

$$
\begin{aligned}
\widetilde{D}^{*} \widetilde{D}= & \nabla^{t *} \nabla^{t}+\frac{1}{4}\left(R-(n-1) H^{2}\right) \\
& -|t|^{2}+2 \sum_{i, j} t_{i j} e^{j} \nabla_{i}+\sum_{i, j} \nabla_{i} t_{i j} e^{j},
\end{aligned}
$$

where $|t|^{2}=\sum_{i, j} t_{i j}^{2}$ is the norm of $\mathcal{T}$.

Theorem 3.2. Let $M \subset N$ be a compact spin hypersurface, and $\lambda$ be the eigenvalue of $\widetilde{D}^{*} \widetilde{D}$ with eigenspinor $\phi$. Then

$$
\lambda \geq \frac{1}{4} \inf _{M}\left(R-(n-1) H^{2}\right)+\inf _{M}\left|Q_{\phi}\right|^{2}
$$

where $\left|Q_{\phi}\right|^{2}=\sum_{i, j} Q_{\phi, i j}^{2}$ and

$$
Q_{\phi, i j}=\frac{1}{2} \mathcal{R} e\left(e^{i} \nabla_{j} \phi+e^{j} \nabla_{i} \phi, \phi /|\phi|^{2}\right)
$$

If $\lambda$ achieves its minimun, then $\left|Q_{\phi}\right|$ is constant. Moreover, either (i) or (ii) holds:

(i) $H=0, \operatorname{tr} Q_{\phi}=$ constant, $R=4\left(\operatorname{tr} Q_{\phi}\right)^{2}-4\left|Q_{\phi}\right|^{2}, \lambda=\left(\operatorname{tr} Q_{\phi}\right)^{2}$ and

$$
R_{i j}=4 \operatorname{tr} Q_{\phi} Q_{\phi, i j}-4 \sum_{k} Q_{\phi, i k} Q_{\phi, k j}+2 \sum_{k} \mathcal{R} e \frac{\left(e^{k} d Q_{\phi, i k} \phi, e^{j} \phi\right)}{|\phi|^{2}}
$$

(ii) $H=$ constant, $\operatorname{tr} Q_{\phi}=0, R=n(n-1) H^{2}-4\left|Q_{\phi}\right|^{2}, \lambda=\frac{(n-1)^{2} H^{2}}{4}$ and

$$
R_{i j}=(n-1) H^{2} \delta_{i j}-4 \sum_{k} Q_{\phi, i k} Q_{\phi, k j}+2 \sum_{k} \mathcal{R} e \frac{\left(e^{k} d Q_{\phi, i k} \phi, e^{j} \phi\right)}{|\phi|^{2}}
$$


Proof. (3.24) implies

$$
\int_{M}|\widetilde{D} \phi|^{2}=\int_{M}\left|\nabla^{t} \phi\right|^{2}+\frac{1}{4}\left(R-(n-1) H^{2}\right)|\phi|^{2}+\left(2 \sum_{i, j} t_{i j} Q_{\phi, i j}-|t|^{2}\right)|\phi|^{2} .
$$

While $t_{i j}=Q_{\phi, i j}, 2 \sum_{i, j} t_{i j} Q_{\phi, i j}-|t|^{2}$ achieves its maximum $|t|^{2}$. Hence the proof of the first part of the theorem is complete.

When $\lambda$ achieves its minimum, $\nabla^{t} \phi=0$. Then

$$
\nabla_{i} \phi=-\sum_{j} Q_{\phi, i j} e^{j} \phi-\frac{H}{2} e^{0} e^{i} \phi, \quad D \phi=\left(\operatorname{tr} Q_{\phi}\right) \phi-\frac{n H}{2} e^{0} \phi .
$$

Obviously, $d|\phi|^{2}=0$. Hence $Q_{\phi}$ is well-defined on $M$. On the other hand,

$$
\begin{aligned}
& \frac{1}{2} \sum_{k} R_{i k} e^{k} \phi=\frac{1}{4} \sum_{j, k, l} R_{i j k l} e^{j} e^{k} e^{l} \phi=\sum_{j, k, l} e^{j}\left(\nabla_{j} \nabla_{i}-\nabla_{i} \nabla_{j}\right) \phi \\
& =-\sum_{j, l} \nabla_{j}\left(Q_{\phi, i l} e^{j} e^{l} \phi\right)-\sum_{j, l} Q_{\phi, i l} e^{j} e^{l} \nabla_{j} \phi-\left(\nabla_{i} \operatorname{tr} Q_{\phi}\right) \phi-\operatorname{tr} Q_{\phi} \nabla_{i} \phi \\
& \quad+\frac{n}{2} \nabla_{i} H e^{0} \phi+\frac{n H}{2} e^{0} \nabla_{i} \phi+\frac{1}{2} \sum_{j} \nabla_{j} H e^{0} e^{j} e^{i} \phi+\frac{H}{2} \sum_{j} e^{0} e^{j} e^{i} \nabla_{j} \phi \\
& =2 \sum_{j} \nabla_{j} Q_{\phi, i j} \phi+\sum_{j, l} \nabla_{j} Q_{\phi, i l} e^{l} e^{j} \phi+2 \sum_{j} Q_{\phi, i j} \nabla_{j} \phi+\sum_{j, l} Q_{\phi, i l} e^{l} e^{j} \nabla_{j} \phi \\
& \quad-\left(\nabla_{i} \operatorname{tr} Q_{\phi}\right) \phi-\operatorname{tr} Q_{\phi} \nabla_{i} \phi+\frac{n-2}{2} \nabla_{i} H e^{0} \phi \\
& \quad+\frac{(n-2) H}{2} e^{0} \nabla_{i} \phi-\frac{1}{2} e^{0} e^{i} d H \phi-\frac{H}{2} e^{0} e^{i} D \phi .
\end{aligned}
$$

And

$$
\begin{aligned}
-\frac{1}{2} R \phi= & \frac{1}{2} \sum_{i, k} R_{i k} e^{i} e^{k} \phi=2 \sum_{j} \nabla_{j} Q_{\phi, i j} e^{i} \phi+2 \sum_{j} Q_{\phi, i j} e^{i} \nabla_{j} \phi-2\left(d \operatorname{tr} Q_{\phi}\right) \phi \\
& -2 \operatorname{tr} Q_{\phi} D \phi-(n-1) e^{0} d H \phi-(n-1) H e^{0} D \phi \\
= & 2 \sum_{j} \nabla_{j} Q_{\phi, i j} e^{i} \phi-2\left(d \operatorname{tr} Q_{\phi}\right) \phi-(n-1) e^{0} d H \phi+H \operatorname{tr} Q_{\phi} e^{0} \phi \\
& +2 \sum_{j} Q_{\phi, i j} e^{i} \nabla_{j} \phi-2\left(\operatorname{tr} Q_{\phi}\right)^{2} \phi-\frac{n(n-1) H^{2}}{2} \phi .
\end{aligned}
$$

Take the real part of inner product of the above equality with $\phi$, we obtain,

$$
R=n(n-1) H^{2}+4\left(\operatorname{tr} Q_{\phi}\right)^{2}-4\left|Q_{\phi}\right|^{2} .
$$

Therefore

$$
2 \sum_{i, j} \nabla_{j} Q_{\phi, i j} e^{i} \phi-2\left(d \operatorname{tr} Q_{\phi}\right) \phi-(n-1) e^{0} d H \phi+H\left(\operatorname{tr} Q_{\phi}\right) e^{0} \phi=0
$$


Take the real part of inner product of above equality with $e^{0} \phi$, we obtain

$$
H \operatorname{tr} Q_{\phi}=0 .
$$

Since we have

$$
\lambda \int_{M}|\phi|^{2}=\int_{M} \frac{1}{4}\left(R-(n-1) H^{2}\right)|\phi|^{2}+\left|Q_{\phi}\right|^{2}|\phi|^{2},
$$

where $\lambda=\frac{1}{4} \inf _{M}\left(R-(n-1) H^{2}\right)+\inf _{M}\left|Q_{\phi}\right|^{2}$. This and (3.29) imply both $\left|Q_{\phi}\right|^{2}$ and $(n-1)^{2} H^{2}+4\left(\operatorname{tr} Q_{\phi}\right)^{2}$ are constants. Hence, by (3.31), we have either (i) $H=0, \operatorname{tr} Q_{\phi}=$ constant; or $(i i) H=$ constant, $\operatorname{tr} Q_{\phi}=0$. In both cases, we have

$$
\sum_{j} \nabla_{j} Q_{\phi, i j}=0
$$

Case $(i) H=0, \operatorname{tr} Q_{\phi}=$ constant: Obviously, we have

$$
R=4\left(\operatorname{tr} Q_{\phi}\right)^{2}-4\left|Q_{\phi}\right|^{2}, \quad \lambda=\left(\operatorname{tr} Q_{\phi}\right)^{2} .
$$

On the other hand,

$$
\begin{aligned}
\frac{1}{2} \sum_{k} R_{i k} e^{k} \phi & =\sum_{j} e^{j} d Q_{\phi, i j} \phi+2 \sum_{j} Q_{\phi, i j} \nabla_{j} \phi+\sum_{j} Q_{\phi, i j} e^{j} D \phi-\operatorname{tr} Q_{\phi} \nabla_{i} \phi \\
& =\sum_{j} e^{j} d Q_{\phi, i j} \phi-2 \sum_{j, k} Q_{\phi, i j} Q_{\phi, j k} e^{k} \phi+2 \operatorname{tr} Q_{\phi} \sum_{k} Q_{\phi, i k} e^{k} \phi .
\end{aligned}
$$

Therefore,

$$
R_{i j}=4 \operatorname{tr} Q_{\phi} Q_{\phi, i j}-4 \sum_{k} Q_{\phi, i k} Q_{\phi, k j}+2 \sum_{k} \mathcal{R} e \frac{\left(e^{k} d Q_{\phi, i k} \phi, e^{j} \phi\right)}{|\phi|^{2}}
$$

Case (ii) $H=$ constant, $\operatorname{tr} Q_{\phi}=0$ : Obviously, we have

$$
R=n(n-1) H^{2}-4\left|Q_{\phi}\right|^{2}, \quad \lambda=\frac{(n-1)^{2} H^{2}}{4} .
$$

On the other hand,

$$
\begin{aligned}
\frac{1}{2} \sum_{k} R_{i k} e^{k} \phi= & \sum_{j} e^{j} d Q_{\phi, i j} \phi+2 \sum_{j} Q_{\phi, i j} \nabla_{j} \phi+\sum_{j} Q_{\phi, i j} e^{j} D \phi \\
& +\frac{(n-2) H}{2} e^{0} \nabla_{i} \phi-\frac{H}{2} e^{0} e^{i} D \phi \\
= & \sum_{j} e^{j} d Q_{\phi, i j} \phi-2 \sum_{j} Q_{\phi, i j} Q_{\phi, j k} e^{k} \phi+\frac{(n-1) H^{2}}{2} e^{i} \phi
\end{aligned}
$$

Therefore,

$$
R_{i j}=(n-1) H^{2} \delta_{i j}-4 \sum_{k} Q_{\phi, i k} Q_{\phi, k j}+2 \sum_{k} \mathcal{R} e \frac{\left(e^{k} d Q_{\phi, i k} \phi, e^{j} \phi\right)}{|\phi|^{2}} .
$$




\section{Acknowledgements}

The author would like to thank Prof. S.T. Yau for his valuable suggestion and continuing encouragement.

\section{References}

[B] H. Baum, Eigenvalue estimates for Dirac operators coupled to instantons, Ann. Global Anal. Geom. 12 (1994), 193-209.

[F1] T. Friedrich, Der erste eigenwert des Dirac-operators einer kompakten, Riemannschen Mannigfaltigkeit nicht negativer skalarkrummung, (German) Math. Nachr. 97 (1980), $117-146$.

[F2] _ On the spinor representation of surfaces in Euclidean 3-space, dg-ga/9712021.

[H] R. Harvey, Spinors and calibrations, Perspectives in Mathematics, 9, Academic Press Inc., Boston, MA, 1990.

[Hi1] O. Hijazi, A conformal lower bounds for the smallest eigenvalue of the Dirac operator and Killing spinors, Comm. Math. Phys. 104 (1986), 151-162.

[Hi2] _ Lower bounds for the eigenvalues of the Dirac operator, J. Geom. Phys. 16(1995), 27-38.

[Hit] N. Hitchin, Harmonic spinors, Adv. Math. 14 (1974), 1-55.

[LM] H. Lawson and M. Michelson, Spin geometry, Princeton Univ. Press. 1989.

[L] A. Lichnerowicz, Spineurs harmoniques, (French) C. R. Acad. Sci. Paris 257 (1963), $7-9$.

[LS] F. Lizzi and R. J. Szabo, Duality symmetries and noncommutative geometry of string spacetime, hep-th/9707202.

[PT] T. Parker and C. Taubes, On Witten's proof of the positive energy theorem, Comm. Math. Phys. 84 (1982), 223-238.

[SY1] R. Schoen and S.T. Yau, On the proof of the positive mass conjecture in general relativity, Comm. Math. Phys. 65 (1979), 45-76.

[SY2] _ Proof of the positive mass theorem II, Comm. Math. Phys. 79 (1981), 231-260.

[S1] L. B. Szabados, Two-dimensional Sen connections in general relativity, Class Quantum. Grav. 11 (1994), 1833-1846.

[S2] _ , Two-dimensional Sen connections and quasi-local energy-momentum, Class Quantum. Grav. 11 (1994), 1847-1866.

[VW] C. Vafa and E. Witten, Eigenvalue inequaltiy for fermious in gauge theories, Comm. Math. Phys. 95 (1984), 257-276.

[W] E. Witten, A new proof of the positive energy theorem, Comm. Math. Phys. 80 (1981), 381-402.

[YZ] S. T. Yau and X. Zhang, On the massive Seiberg-Witten theory, preprint.

[Z] X. Zhang, Positive mass conjecture for five dimensional Lorentzian manifolds, preprint.

Morningside Center of Mathematics, Institute of Mathematics, Chinese Academy of Sciences, Beijing 100080, CHINA

E-mail address: xzhang@math08.math.ac.cn 